\title{
Alimentación saludable en la formación de estudiantes de carreras del área pedagógica
}

\author{
Teaching healthy diet concepts during \\ teacher training period
}

\begin{abstract}
Malnutrition by excess presented in the child-young population must be treated by a multidisciplinary team in which doctors, family and school, but specially teachers should play an essential role. Regarding this situation, student teachers need to acquire knowledge about healthy diet concepts during their training period in order to achieve this role. The aim of this research was to interpret the importance that students training to become teachers give to a healthy diet. For this purpose, a qualitative, phenomenological research was carried out with a sample consisting of 40 university students from different areas related to teaching. The data was collected using a semistructured interview and the answers were analyzed with the content analysis methodology. The results showed that student teachers think it is necessary to have a healthy diet during their training period. However, this is not followed due to different circumstances. Student teachers who will work with elementary school children expressed that their study programs should include elective subjects related with healthy diet habits. This situation is not equally perceived by student teachers who will work with teenagers. They believe these contents are for selfcare. It is concluded that student teachers who will work with teenagers do not perceive they play an education role concerning healthy diet habits. They do not consider the elective subjects related to healthy diet habits useful for preventing malnutrition by excess among children and teenagers.
\end{abstract}

Key words: student teachers, teaching, malnutrition by excess, qualitative research.

\section{INTRODUCCIÓN}

La presencia de malnutrición por exceso es uno de los problemas de salud pública que presenta la población infantil y juvenil, situación que se produce en números países y que es identificada por la Organización Mundial de la Salud. La presencia de estas patologías puede relacionarse con condiciones fetales, aspectos dietéticos, sedentarismo, características socioeconómicas y/o nivel educacional de la población de diversos grupos etarios (1-3).

En los últimos años, se han concentrado esfuerzos en mejorar la calidad de vida y salud de menores en edad escolar a través de diversos programas, especialmente de educación, destinados a formar o reforzar hábitos saludables de alimentación y de vida en esta población. Si bien, estos proyectos presentan logros positivos, tienden a no mantenerse en el tiempo o ser muy locales, por lo que la obesidad y sobrepeso
Claudia Troncoso P. (1)

Carlos Doepking M. (2)

Carolina Zúñiga B. (2)

(1) Departamento de Ciencias Clínicas y Preclínicas. Carrera de Nutrición y Dietética. Facultad de Medicina. Universidad Católica de la Santísima Concepción, Concepción, Chile. (2) Carrera de Nutrición y Dietética. Facultad de Medicina. Universidad Católica de la Santísima Concepción, Concepción, Chile.

Dirigir la correspondencia a: Profesora

Claudia Troncoso Pantoja Facultad de Medicina

Universidad Católica de la Santísima Concepción. Campus San Andrés. Alonso de Ribera 2850. Concepción. Chile. Fonos: 56-041-2345427-56-041-2345406 E-mail: ctroncosop@ucsc.cl

Este trabajo fue recibido el 14 de Agosto de 2012 y aceptado para ser publicado el 6 de Diciembre de 2012. crece en forma exponencial en estos niños (4-7).

Entre los agentes sociales que se identifican en la formación de conductas de vida sana en niños y adolescentes están la familia, en especial la imagen materna y la escuela. En esta última, los docentes juegan un papel preponderante en la entrega de conocimientos y conductas de vida saludable. Sin embargo, se ha identificado que la información que reciben los estudiantes del área de pedagogía en su formación curricular es en ocasiones insuficiente para modificar hábitos de vida como los alimentarios (8-13).

Frente a este escenario, existen políticas públicas orientadas a crear ambientes sanos en algunas instituciones de educación superior y de esta manera fortalecer la formación de conductas saludables en los futuros docentes, a través de programas como el denominado: "Universidades Saludables". Esta política de salud incluye una serie de acciones que incorporan 
actividades de promoción en el ambiente laboral y estudiantil, teniendo como meta, especialmente para los estudiantes, el ser reflejos de conductas de vida saludable en sus familias, entornos laborales y en la población en general $(14,15)$.

La trascendencia que tiene la formación o el reforzamiento de conductas de vida saludables, orientadas especialmente a una alimentación sana, en agentes claves en la formación de hábitos de vida en niños y adolescentes como son los pedagogos es la base de esta investigación, que tiene como objetivo el interpretar la percepción de la importancia de una alimentación saludable en estudiantes de carreras del área educacional.

\section{SUJETOS Y MÉTODO}

Para responder los objetivos de la investigación, se utilizó un diseño cualitativo con enfoque fenomenológico, en donde los sujetos en investigación corresponden a estudiantes de educación superior que pertenecen a una de las carreras de pedagogía que oferta el establecimiento universitario: Educación de Párvulos; Pedagogía en Educación Diferencial; Pedagogía Educación General Básica; Pedagogía en Educación Media en Matemáticas; Pedagogía Media en Religión y Educación Moral; Pedagogía Educación Media en Biología y Ciencias Naturales y Pedagogía Educación Media en Lenguaje y Comunicación.

Para ingresar a la investigación, los alumnos debían ser alumnos regulares de alguna carrera de pedagogía impartida en la Facultad de Educación del Campus San Andrés, Universidad Católica de la Santísima Concepción (UCSC), Concepción, Chile. Además una edad entre 18 años y 30 años y firmar el consentimiento informado, en donde se les indicaba los objetivos y metodología de la investigación. Esta última actividad es la forma de controlar los aspectos éticos del estudio.

El tamaño muestral se obtuvo a través del punto de saturación, esto es, una vez que las respuestas comienzan a coincidir entre los entrevistados. La muestra se completó con 40 entrevistas. De estas últimas, 18 se realizaron en estudiantes de carreras del primer ciclo básico: Educación de Párvulos; Pedagogía en Educación Diferencial; Pedagogía Educación General Básica; las entrevistas restantes se realizaron en estudiantes de Pedagogía en Educación Media en Matemáticas; Pedagogía Media en Religión y Educación Moral; Pedagogía Educación Media en Biología y Ciencias Naturales y Pedagogía Educación Media en Lenguaje y Comunicación.

La recolección de la información se realizó a través de entrevistas individuales semiestructurada, en donde se profundizó sobre la percepción de los estudiantes de pedagogía sobre el significado de alimentación saludable, la trascendencia que presenta la alimentación saludable en su formación académica y la necesidad que tiene en su proyección profesional la alimentación sana. Esta entrevista fue realizada por dos estudiantes de la carrera de Nutrición y Dietética de la Facultad de Medicina de la UCSC, capacitados para esta actividad, los que transcribieron las entrevistas a un formato papel para ser analizadas por el investigador principal. Este análisis se realizó utilizando la técnica de análisis de contenido.

Para realizar el análisis, se separaron las entrevistas entre aquellos alumnos que estudiaban carreras de pedagogía orientadas a los preescolares (2-5 años) y primer ciclo educacional (educación básica), que incluye a menores entre 6 a 13 años y segundo ciclo educacional (educación media), estudiantes que desempeñarán competencias profesionales con adolescentes entre 14 a 17 años de edad.

\section{RESULTADOS}

1.- Significado de alimentación saludable para estudiantes de carreras de pedagogía.

Se presenta en gran parte de los entrevistados la interpretación de asociar el concepto de "alimentación saludable" al consumo de una dieta balanceada, entendiendo este último concepto como una alimentación que presente un bajo aporte de alimentos de alta densidad energética y una alta concentración de fibra, identificando claramente la importancia de la ingesta de frutas y verduras en la dieta habitual, además de agua. Otros nutrientes como proteínas u oligoelementos, como el sodio, son identificados también como parte de una dieta equilibrada. Esta percepción se presenta independiente de la carrera de pedagogía que estudian los entrevistados.

"Comer, cosas con pocas calorías, comer frutas, verduras, cosas que realmente te alimenten" [Estudiante 27].

En algunos de los entrevistados, además de la calidad nutritiva que presenta su dieta, se identifica como parte de la alimentación saludable, el realizar un adecuado patrón alimentario, entendiendo esto último como horarios de alimentación establecidos (tres a cuatro comidas durante el día).

"Primero que nada comer sano, frutas, verduras, comer a las horas y no comer a deshora o dulces, eso" [Estudiante 9].

Sin embargo, aunque se presenta en gran parte de los entrevistados la percepción de la importancia que presenta una alimentación saludable, esta conducta la realiza sólo una minoría de estudiantes, especialmente si presentan algún tipo de patología gástrica y no por opción de estilo de vida.

Se identifican numerosas motivaciones que son codificadas por los entrevistados como "dificultades" para realizar una dieta sana, como: la facilidad de conseguir en el establecimiento educacional o en sus alrededores, alimentos de bajo costo que presentan alta densidad energética; las preferencias personales de los estudiantes, en donde se privilegia el mejor valor sápido que presentan los alimentos con una alto aporte de grasas, en desmedro de otros como frutas y verduras; la falta de disponibilidad de tiempo durante la jornada académica que facilita la adquisición de alimentos de alta densidad energética en la universidad o a no realizar algunos horarios de alimentación, como el desayuno o almuerzo.

"No, porque a mi igual me gusta la comida chatarra y por los tiempos porque cuando uno tiene 10 minutos no alcanza a comer o en el almuerzo no alcanza a comer algo saludable, como por ejemplo legumbres. Siempre se compra lo más fácil o al comprarse un paquete de papas fritas o una bebidas, no te cubren el hambre sino que uno come algo que te quitan el hambre y que sean cosas ricas" [Estudiante 16].

Sólo un entrevistado interpretó que para el ejercicio de su futura profesión es necesario realizar una dieta saludable, percibiendo que de esta manera logrará un mejor proceso de aprendizaje en sus estudiantes.

"Sí, yo cuido mi alimentación porque a mí no me gustaría que mis alumnos digieran la profesora nos enseña a nosotros que tenemos que comer sano y ella no practica lo que nos está enseñando a nosotros, intento comer comida a las horas, no como comida chatarra, me preocupo de tomar agua diariamente y cosas así" [Estudiante 19].

\section{2.- Conceptos de alimentación saludable en la formación} académica de estudiantes de carreras de pedagogía.

Existió una marcada diferencia de opinión respecto a incluir en sus programas de formación temas de alimentación saludable entre los entrevistados que estudian carreras de pedagogía dirigidas al primer ciclo de educación escolar y pre- 
escolar (menores entre 2 a 13 años) con aquellos estudiantes que ejercerán su profesión con estudiantes del segundo ciclo de educación (14 a 17 años).

En los futuros docentes del primer ciclo, existe una percepción positiva de la importancia que tendrán en la formación de hábitos de vida saludable en los menores, incluyendo la alimentación sana, identificando claramente su rol de educadores de conductas alimentarias saludables, en el grupo etario en donde desarrollarán sus competencias profesionales en el aula y su trascendencia en la actual situación de salud del país, debido a la gran prevalencia de malnutrición por exceso de la población preescolar y escolar.

"Yo creo que sí, porque nosotros nos encargamos de educar a los niños y también tiene que ir involucrado" [Estudiante 8].

Esta situación no es extrapolable a los estudiantes del segundo ciclo de educación. Si bien se presentan futuros educadores que identifican la importancia de recibir en su formación profesional contenidos necesarios para aplicar en el aula la formación de hábitos de alimentación saludables, un grupo importante de entrevistados los codifica sólo como parte de su autocuidado, mencionando algunos que estos contenidos serían innecesarios en el desarrollo de sus competencias profesionales.

"Nosotros no creo que tanto, yo creo que más lo de básica que trabajan más con niños y pueden ir formando desde más pequeños, pero nosotros que somos más grandes no. Creo que debería ser un ramo optativo una cosa así y al que le interese aprender de eso puede ser que sí, pero no tanto como de malla" [Estudiante 6].

Una situación en común que se presenta en los entrevistados que identifican positivamente la importancia de recibir contenidos para formar en hábitos alimentarios saludables, es la ubicación de estos en su malla curricular, ya que la mayoría los percibe necesarios en una asignatura de carácter optativo y no como parte del curriculum mínimo de su formación profesional.

\section{3.- Alimentación saludable en la proyección profesional de estudiantes de carreras de pedagogía.}

Para gran parte de los entrevistados, independiente del ciclo educacional en el que ejercerán las competencias profesionales, se presenta la codificación que los contenidos de alimentación saludable serán utilizados en su futuro profesional.

La diferencia se concentra en que gran parte de los estudiantes que trabajarán con niños menores de 13 años, los que perciben que estos últimos deben recibir información de hábitos de alimentación saludable, para fortalecer o incorporar estos contenidos que deben ser recibidos en sus hogares y porque identificarían la presencia de malnutrición por exceso en este grupo etario y la necesidad que presenta su intervención en la prevención y tratamiento de esta patología crónica.

"Sí, yo creo que en algún momento sí, porque yo que creo que hay muchos niños que no se les enseñan en la casa entonces, la mayoría del tiempo o la mitad de su tiempo la van a pasar en el colegio, entonces uno también tiene que tratar de abarcar muchas cosas aparte de las materias del colegio" [Estudiante 28].

En cambio, la mayoría de los entrevistados que trabajarán con adolescentes, identifican la incorporación de contenidos de alimentación saludable en su malla curricular, pero destinada a mejorar su calidad de vida, más que para modificar conductas alimentarias en sus futuros estudiantes.

"Sí, obviamente, sino podría tener alguna enfermedad y no podría cumplir mi la labor como debería" [Estudiante 13].

No obstante a lo anterior, se identificó que algunos de los estudiantes universitarios que trabajarán con menores del segundo ciclo de educación, perciben la presencia de patologías que se asocian a la alimentación, como obesidad, anorexia nervosa o bulimia y que por lo tanto, necesitan contenidos de alimentación sana para prevenir estas patologías. Sin embargo, esta percepción fue en una minoría de los entrevistados.

"Sí, claro. Hoy en día hay mucha obesidad y se nota que los niños no son bien educados" [Estudiante 25].

\section{DISCUSIÓN}

Existe evidencia suficiente respecto a que actividades de intervención en educación y salud realizadas en niños y adolescentes en el ámbito escolar, permiten mejorar o incorporar algunas conductas saludables, en especial, si en estas actividades han participado otros agentes de formación de hábitos en estos menores, como son su familia o docentes (16-18). Sin embargo, entre las conductas saludables, la alimentación en las escuelas no se caracteriza por ser cualitativa y cuantitativamente equilibrada para cubrir las necesidades nutricionales de los niños y adolescentes, surgiendo la inquietud del rol que presentan las escuelas y otras entidades en la formación de hábitos alimentarios saludables (19-23).

Ante esta situación, el papel de los pedagogos es esencial, sin embargo, en su etapa de formación y como ocurre en la mayoría de los estudiantes de educación superior, no se presentan conductas alimentarias sanas $(24,25)$.

La mala alimentación es frecuente en los alumnos universitarios. Una gran cantidad de estudios ha mencionado esta situación, identificando como características alimentarias el consumo de alimentos de alta densidad energética y limitando la ingesta de alimentos como frutas y verduras, en donde la carrera que cursan no haría diferencia frente a esta situación (26-35). Este último hecho se presenta en esta investigación, en que los estudiantes de pedagogía identifican con claridad que deben realizar una alimentación que codifican como sana, pero que en la práctica no es seguida por ellos, debido a diversas circunstancias como los costos de alimentos, la falta de oferta de alimentos en el centro educacional y la inadecuada selección de estos últimos que los estudiantes realizan.

Sin embargo, se presenta una diferencia de los entrevistados en la percepción de la importancia de incluir contenidos de alimentación saludable en su formación profesional. Aquellos que se forman para educar a niños del primer ciclo escolar o preescolar refieren que este tema es de necesidad para su formación profesional, ya que será necesario para educar a los menores que formarán en hábitos alimentarios saludables y que por lo tanto, estos contenidos deberían presentarse en la malla curricular de sus respectivas carreras, aunque lo consideran como un optativo, sin ser parte de los contenidos mínimos para su formación.

Esta situación no se explicita de esta manera en los estudiantes que proyectarán su futuro laboral en la formación educacional con adolescentes, ya que si bien creen que son necesario recibir formación en alimentación saludable, esta la codifican para su autocuidado más que para el reforzamiento de hábitos sanos. Al igual que los estudiantes del primer ciclo de educación, lo consideran como asignatura de carácter optativo.

\section{CONCLUSIÓN}

Los estudiantes de pedagogía perciben que no realizan una alimentación saludable en el establecimiento universitario, 
mencionando diversos motivos para la falta de adherencia a una dieta sana, como la falta de recursos económicos o una inadecuada selección de los alimentos a consumir.

En los entrevistados, aquellos que trabajarán con niños preescolares o escolares identifican su rol en la formación de hábitos alimentarios sanos en sus educandos. Por lo tanto, interpretan la necesidad de recibir en sus planes de formación profesional estos contenidos, pero en asignaturas de carácter optativo. En cambio, los estudiantes de pedagogía que ejercerán su profesión con adolescentes, consideran que deben recibir esta formación, pero codificando que estos contenidos son necesarios para su autocuidado y no para reforzar hábitos alimentarios saludables en sus pupilos.

Esta situación debe ser considerada por los equipos médicos frente a la actual situación epidemiológica que presenta la población infanto-juvenil, en donde se destaca la presencia de malnutrición por exceso. Si bien la formación en hábitos de alimentación saludable no es exclusiva de los centros educacionales, estos cada vez tienen un rol más protagónico. Si los futuros educadores de menores, especialmente aquellos que forman en la etapa juvenil, no presentan claridad en su rol de reforzamiento de conductas alimentarias sanas, será dificultoso mejorar la calidad de vida de la población, lo que puede repercutir en la mantención de las actuales cifras de malnutrición por exceso que presenta el país.

Es importante mencionar la percepción positiva que presentan los entrevistados del primer y segundo ciclo de formación educacional sobre la necesidad de incluir contenidos de hábitos de vida saludable, específicamente de alimentación, en sus mallas de formación profesional. No obstante, sólo los consideran para ser entregados en asignaturas de carácter optativo, por lo tanto, esta formación será una competencia de aquellos estudiantes que "seleccionen" adquirir conocimientos en el tema, limitando la formación de conductas de vida sana a sus futuros alumnos y favoreciendo de esta medida la presencia de sobrepeso y obesidad en la población infantil y juvenil. Como refiere Bayona (36) en trabajo realizado en estudiantes de una carrera del área médica: "... No se puede hacer promoción de algo que no se conoce o no se practica".

\section{RESUMEN}

La malnutrición por exceso que se presenta en la población infanto-juvenil debe ser tratada a través de un equipo multidisciplinar, en el que además del cuerpo médico, la familia y la escuela, en especial los pedagogos, presentan un rol fundamental. Frente a esta situación es necesaria la formación de estos profesionales en la etapa universitaria en temas de alimentación saludable para cumplir con esta función.

El objetivo de la investigación fue interpretar la percepción de la importancia de una alimentación saludable en estudiantes de carreras del área educacional. Para cumplir esta meta se utilizó un estudio cualitativo con enfoque fenomenológico, en una muestra de 40 estudiantes universitarios de carreras del área pedagógica que cumplían con los criterios de selección de la investigación. Para recolectar información se utilizó una entrevista semi-estructurada y las respuestas se analizaron a través de análisis de contenido. Los estudiantes identificaron que es necesario realizar una alimentación sana durante su etapa de formación profesional, sin embargo, por diversas circunstancias no es desarrollada por los entrevistados. Los estudiantes que ejercerán sus competencias profesionales con menores de primer ciclo educacional codifican que sus programas de estudios deben incluir contenidos de alimen- tación saludable en asignaturas optativas, ya que lo utilizarán en su futuro laboral. Esta situación no es percibida por los estudiantes que trabajarán con adolescentes, ya que identifican que estos contenidos son necesarios para su autocuidado. Se concluye que los estudiantes de pedagogía que desarrollarán su futuro profesional con adolescentes no perciben su rol en la formación de hábitos alimentarios sanos. El carácter de optativo que identifican para alguna asignatura que incluya contenidos de alimentación saludable, no favorecen a la prevención o tratamiento de la malnutrición por exceso de niños y adolescentes.

Palabras clave: Estudiantes; pedagogía; malnutrición por exceso; estudio cualitativo.

\section{BIBLIOGRAFÍA}

1. Marín V.; Rodríguez L.; Buscaglione R. et al. Programa MINSAL-FONASA para el tratamiento de la obesidad infanto-juvenil. Rev Chil Pediatr 2011; 82(1): 21-8.

2. Aguilar M.; González E.; García C. et al. Estudio comparativo de la eficiencia del Indice de Masa Corporal y el porcentaje de grasa corporal como métodos para el diagnóstico de sobrepeso y obesidad en población pediátrica. Nutr Hosp 2012; 27(1): 185-91.

3. Kain J.; Vio F.; Albala C. Tendencias en la obesidad y factores determinantes en América Latina. Cad Saúde Pública 2003. 19 (suppl. 1): S77-S86.

4. Pérez L.; Bayona I.; Mingo T. et al. Utilidad de los programas de educación nutricional para prevenir la obesidad infantil a través de un estudio piloto en Soria. Nutr Hosp 2011; 26(5): 1161-7.

5. Vío F.; Zacarías I.; Lera L. et al. Prevención de la obesidad en escuelas básicas de Peñalolén: componente alimentación y nutrición. Rev Chil Nutr 2011;38(3): 268-73.

6. Barrio I.; Ayudarte M.; García M. et al. Presencia de las actuales prioridades de salud infantil y adolescente en los libros de textos escolares. Gac Sanit 2008; 22(3):227-31.

7. Ibarra J.; Alarcón M. Malnutrición por exceso en escolares. Rev Chil Pediatr 2010;81(6): 506-14.

8. Unicef. Formación de hábitos de vida saludable. [online] [citado mayo 2012]. Disponible en www: http://www. unicef.org/venezuela/spanish/educinic9.pdf.

9. Visser R. Plan de acción holístico contra el sobrepeso y la obesidad en niños en Aruba. Rev Cubana Salud Pública. 2005; 31(4):353-60.

10. Flores S.; Klünder M.; Medina P. La escuela primaria como ámbito de oportunidad para prevenor el sobrepeso y obesidad en los niños. Bol Med Hosp Infant Mex 2008; 65(6):626-38.

11. Rodríguez N. Retos de la formación de docentes en Venezuela. Rev Pediatr 2003; 25(73): 3-12.

12. Restrepo S.; Maya M. La familia y su papel en la formación de los hábitos alimentarios en el escolar. Un acercamiento a la cotidianidad. Bol Antropol 2005; 19(36): 127-48.

13. Llargués E.; Franco R.; Recasens A. et al. Estado ponderal, hábitos alimentarios y de actividad física en escolares de primer curso de educación primaria: estudio Avall. Endocrinol Nutr 2009; 56(06): 287-92.

14. Meda R.; De Santos F.; Lara B. et al. Evaluación de la percepción de la calidad de vida y el estilo de vida en estudiantes desde el contexto de las universidades promotoras de la salud. Rev Educ Des. 2008;5-16.

15. Vio F.; Salinas J. Promoción de salud y calidad de vida en Chile: Una política con nuevos desafíos. Rev Chil Nutr 2006; 33(11):252-9. 
16. Ibarra J.; Alarcón M. Malnutrición por exceso en escolares. Rev Chil Pediatr 2010; 81(6): 506-14.

17. Botelho L.; Zanirati V.; Paula D. et al. Promotion of a healthy nutrition for school children: learning and perceptions of an operative group. Nutrire Rev Soc Bras Aliment Nutr 2010; 35(2): 103-16.

18. Del Campo M.; Messler V.; Navarro A. Educación alimentario nutricional (EAN) en la enseñanza municipal de Córdoba: una experiencia en investigación-acción participativa (IAP) año 2008. Diaeta (B. Aires) 2010; 28(132): 15-22.

19. Velandia N.; Cárdenas L.; Agudelo N. ¿Qué ofrecen las tiendas escolares y qué consumen los estudiantes durante el descanso?: estudio en la ciudad de Tunja, 2009. Hacia la Promoción de la Salud 2011; 16(1): 99-109.

20. Gabriel C.; Vasconcelos F.; Andrade D. et al. Primera ley de regulación de las cantinas escolares en Brasil: evaluación después de siete años de aplicación. Arch Latinoam Nutr 2009; 59(2)128-38.

21. Román V.; Quintana M. Nivel de influencia de los medios de comunicación sobre la alimentación saludable en adolescentes de colegios públicos de un distrito de Lima. An Fac Med (Perú) 2010; 71(3): 185-9.

22. Kruger N.; Whitacre C. Efecto de la publicidad televisiva sobre la selección de alimentos por escolares del Noreste argentino. Diaeta (B. Aires) 2009; 27(127): 24-33.

23. Loubat $M$. Conductas alimentarias: un factor de riesgo en la adolescencia: resultados preliminares en base a grupos focales con adolescentes y profesores. Ter Psicol 2006;24(1): 31-7.

24. Bollat P.; Durá T.; Gallinas F. Hábitos alimentarios en una población universitaria. Rev Esp Nutr comunitaria 2009; 15(1): 23-8.

25. Souza L.; Malta M.; Donato P. et al. Application of dietary reference intakes in dietary intake assessment of female universities healthcare students in Botucatu, State of Sao Paulo, Brazil. Nutrire Rev Soc Bras Aliment Nutr 2010; 35(3)dez.
26. Vásquez M.; Witriw A.; Reyes C. Estudio preliminar sobre la ingesta alimentaria en estudiantes universitarios de las carreras de medicina y arquitectura de la Universidad de Buenos Aires. Diaeta (B. Aires) 2010; 28(131):14-7.

27. Alba L. Perfil de riesgo en estudiantes de medicina de la Pontifica Universidad Javeriana. Univ Med 2009; 50(2): 143-55.

28. MacMillan N. Valoración de hábitos de alimentación, actividad física y condición nutricional en estudiantes de la Pontificia Universidad Católica de Valparaíso. Rev Chil Nutr 2007; 34(4):330-6.

29. Vargas M.; Becerra F.; Prieto E. Evaluación de la ingesta dietética en estudiantes universitarios. Bogotá, Colombia. Rev Salud Pública 2010; 12(1): 116-25.

30. Pérez N.; Moya M.; Bauce G. Patrones alimentarios y niveles de actividad física en estudiantes de la Universidad Central de Venezuela. Rev Esp Nutr Comunitaria 2009; 15(3): 129-44.

31. Espinoza L.; Rodríguez F.; Gálvez J. et al. Hábitos de alimentación y actividad física en estudiantes universitarios. Rev Chil Nutr 2011; 38(4): 458-65.

32. Troncoso C.; Amaya J. Factores sociales en las conductas alimentarias de estudiantes universitarios. Rev Chil Nutr 2009; 36(4): 1090-7.

33. Tobar L.; Vásquez S.; Bautista L. Descripción de hábitos y comportamientos alimentarios de los estudiantes de la Facultad de Ciencias de la Pontificia Universidad Javeriana. Univ Sci; 2008; 13(1): 55-63.

34. Saad C.; Ibañez E.; León C. et al. Cambios en los hábitos alimentarios de los estudiantes de enfermería de la Universidad El Bosque durante su proceso de formación académica, Bogotá; D.C., 2007. Rev Colom Enferm 2008; 3(3): 51-60.

35. Rivera M. Hábitos alimentarios en estudiantes de la Universidad Juárez Autónoma de Tabasco. Rev Cubana Salud Pública, 2006; 32(3):0.

36. Bayona l.; Navas F.; Fernández F. et al. Hábitos dietéticos en estudiantes de fisioterapia. Nutr Hosp 2007; 22(5):573-7. 